\title{
Visible Light Communication with Input-Dependent Noise: Channel Estimation, Optimal Receiver Design and Performance Analysis
}

\author{
Maysa Yaseen, Malek Alsmadi, Ayse E. Canbilen and Salama Ikki
}

\begin{abstract}
In this paper, single-input single-output (SISO) visible light communication (VLC) subject to signal-dependent shot noise (SDSN) is investigated. We discuss both channel estimation and data transmission. In the former, we introduce least square (LS) and maximum likelihood (ML) estimators. Moreover, we derive the Cramér-Rao lower bound (CRLB) of the channel estimation error. With regard to data transmission, we present optimal and sub-optimal receiver designs and discuss their bit error rate (BER) performance. In particular, a closed-form expression of the BER is derived for a sub-optimal receiver using the on-off-keying (OOK) modulation technique. An approximated expression is then derived for the optimal receiver. Our analysis shows that the CRLB performance does not have a linear relation with the SDSN, thermal noise, or the fading channel. On the other hand, the SDSN has a dramatic effect on the channel estimation error bound, and it can degrade the BER performance. Increased performance degradation can also be noticed due to the joint effects of the channel estimation error and SDSN.
\end{abstract}

Index Terms-Visible light communication, input-dependent noise, channel estimation, receiver design, Cramér-Rao lower bound, least square estimation, maximum likelihood estimation, error performance analysis.

\section{INTRODUCTION}

Indoor illumination is currently undergoing a revolution due to the widespread deployment of light-emitting diodes (LEDs). Unlike older illumination technologies, LEDs are capable of rapidly varying their light intensities. The change is actually too fast to be visible to the human eye, allowing data to be encoded in and transmitted through the emitted light. A photodetector (also known as a photodiode) or an image sensor (i.e. a matrix of photodiodes) can receive the modulated signals and decode the data. Therefore, LEDs can be utilized not only for illumination but also for communication. This functionality has given rise to visible light communication (VLC) technology, where LEDs are used for high-speed data transfer ( 100 Mbps in IEEE 802.15.7 standard and up to multiple Gbps in research) [1].

The use of light propagation for data transmission, whether in terrestrial or satellite applications, can be traced back decades ago. However, with the ever-increasing demand for high data rate wireless communication, VLC has recently regained considerable interest from both industry and academia

Maysa Yaseen, Malek Alsmadi and Salama Ikki are with Lakehead University, ON, Cananda. Emails:\{myaseen, malsamdi, sikki\}@lakeheadu.ca Ayse E. Canbilen is with Konya Technical University, Konya, Turkey, Email: aecanbilen@ktun.edu.tr.
[2]. VLC has many advantages, such as worldwide availability, high security and immunity to radio frequency (RF) interference [3].

Additionally, the visible light spectrum is unlicensed and still substantially unused for communication. Transitioning to this spectrum would allow for low-cost broadband communication while mitigating spectrum density in the RF. VLC systems have relatively simple and inexpensive front-end components for both transmitters and receivers that operate in the baseband. Furthermore, they do not require frequency mixers or complicated algorithms to correct hardware impairments such as phase noise and in-phase/quadrature-phase (I/Q) imbalance [4]. Without a doubt, VLC is a promising candidate for short-range wireless communication in the future technologies.

\section{A. Related Works}

The novel transmission paradigm of VLC is quite different from RF communication. The essential difference is that the transmitted signals in VLC must be real and positive. A reliable VLC system is also required to be flicker-free and satisfy specific lighting and power constraints [5]. Hence, the specifications of VLC requires re-engineering the designs across many layers, including physical layer (PHY) signal processing and modulations [6]. Considering this, advanced PHY techniques originally proposed for RF communication have been reconfigured for use in the context of VLC. For instance, the orthogonal frequency division multiplexing (OFDM) technique has been applied to VLC with certain adjustments [7]-[9].

Afterwards, advanced spatial modulation-based techniques were extended to VLC [10], [11]. Taking the power and lighting constraints into account, constellation designs were revised for multi-carrier VLC systems with the aim of maximizing the minimum distance between two arbitrary constellation symbols [12], [13]. Even more, a framework was developed for LED-based VLC systems for the transmission power and rate optimization [14]. The performance of VLC systems, adopting hybrid modulation techniques, is also investigated under dynamic fading channels [15].

In comparison to RF-based wireless communications, there has been limited consideration of channel estimation for VLC. However, this is a key aspect to investigate when it comes to the synchronization, equalization and implementation of transceiver functions (e.g. precoding at the transmitter and 
data detection at the receiver). Although VLC channels have unique properties that attract a lot of attention during the study of channel estimation, the principles of traditional estimation technologies, such as the pilot-aided schemes, may also apply to VLC scenarios. Considering this, several techniques have been proposed to enhance the channel estimation performance of VLC systems as well as reduce their bit error rate (BER) [16]-[22]. For instance, an evaluation of two channel estimation methods, the least squares (LS) and minimum mean square error (MMSE) algorithms, for indoor OFDM-based VLC systems was presented in [16].

Indeed, channel estimation schemes for OFDM-based VLC systems and their performance are extensively studied in the literature [18]. In addition, [20] proposes a novel neural network based methodology for the prediction of VLC channel parameters. The problem associated with finding a scheme that minimizes the noise variance under maximum and average power constraints was solved in [23]. Next, the BER performance of spatial multiplexing in a multiple-input multiple-output (MIMO) VLC system was evaluated in [24], where the channel matrix, estimated using constructed codes, was used in data detection.

\section{B. Motivation}

The indoor channel characteristics of VLC systems are quite distinct. The channel is effectively more intense due to the line-of-sight (LoS) and non-line-of-sight (NLoS) optical multipath reflections coming from the walls and ceilings that compose an indoor environment. Moreover, the position, shape, and size of the opaque physical obstacles have severe effects on the VLC channel characteristics.

Taking these into careful consideration, the statistical nature of the VLC channel was modelled in [25] using a monotonic decreasing distribution with additive noise corrupting the channel. Notwithstanding that, none of the aforementioned channel estimation studies considered this statistical channel model for a VLC system since it poses major challenges to the provision of reliable data transmission. Up until now, the channel model in [25] was considered only in [26], where the performance of an indoor single-input single-output (SISO) VLC system was evaluated with imperfect channel state information (CSI) at the receiver.

On the other hand, the existence of signal-dependent noise is also a key PHY feature that differentiates VLC from RF for certain receiving devices [27]. Typically, signal-dependent distortions are observed in high signal-to-noise ratio (SNR) VLC links that employ high-gain narrow field-of-view photon avalanche diode (APD) receivers [28]. The photodetector shot noise, which, considering the increasing number of received photons, can be approximated using a Gaussian process, is also signal-dependent due to the quantum nature of the received optical energy [29], [30].

It is widely known that the optimal version of many designs no longer supports signal-dependent shot noise (SDSN), leading to a dramatic deterioration in the system performance along with increased background noises [30], [31]. Thus, the necessity of re-investigating the design structures when SDSN plays a non-negligible role is highlighted in [31], where effective modulation design techniques were proposed for both single and multi-carrier VLC systems. Meanwhile, the mutual information and its lower bound were analyzed for spatial modulation-based VLC in the presence of input-dependent noise in [32].

Following these, efficient transceiver design methods were studied in [33]. The theoretical expression of the BER was obtained in [34], and channel capacity bounds were derived in [35] for VLC systems with signal-dependent Gaussian noise. However, SDSN leads to analytical complications when it comes to deriving closed-form expressions, adding to the already existing challenges to signal processing and modulation design. Therefore, SDSN models have not been sufficiently studied in the VLC field yet.

The lack of studies concerning VLC systems under the joint impacts of SDSN and imperfect CSI motivates our work. This paper fills in the gap by analytically evaluating the error performance of an indoor VLC system with a single LED at the transmitter side and a single photodiode at the receiver side. We consider the monotonic decreasing distribution model for the channel gain as given in [25]. Specifically, an optimal receiver is proposed and compared to a sub-optimal one. Moreover, the VLC channel gains are estimated by implementing two different methods, namely LS and maximum likelihood (ML). Applying the Cramér-Rao lower bound (CRLB) provided a correct estimation of CSI.

\section{Contributions}

Compared to the existing literature and motivated by the significance of the previous reasons, the contribution of this paper can be summarized as follows:

1) The performance of the SISO-VLC system in the presence of SDSN is studied. It is shown that SDSN can degrade the BER performance and has a dramatic effect on the channel estimation error bound.

2) The CRLB that can be used as a benchmark to evaluate the unbiased estimators' efficiency when the system under the effects of SDSN is derived.

3) LS and ML estimators are proposed and their performance is compared with the derived CRLB.

4) An optimal receiver design that can properly treat the SDSN is proposed, and its performance is compared with the traditional ML one. More specifically, we derive a closed-form expression of the sub-optimal receiver BER and an approximated expression of the BER for the optimal one.

5) We studied the effect of imperfect CSI on the system performance. In particular, the error performance in the presence of imperfect CSI has been obtained and analyzed.

Paper Organization. The rest of the paper is organized as follows: Section II describes the channel and system models. Next, Section III discusses the channel estimation and the Cramér-Rao lower bound. Then, Section IV proposes the 


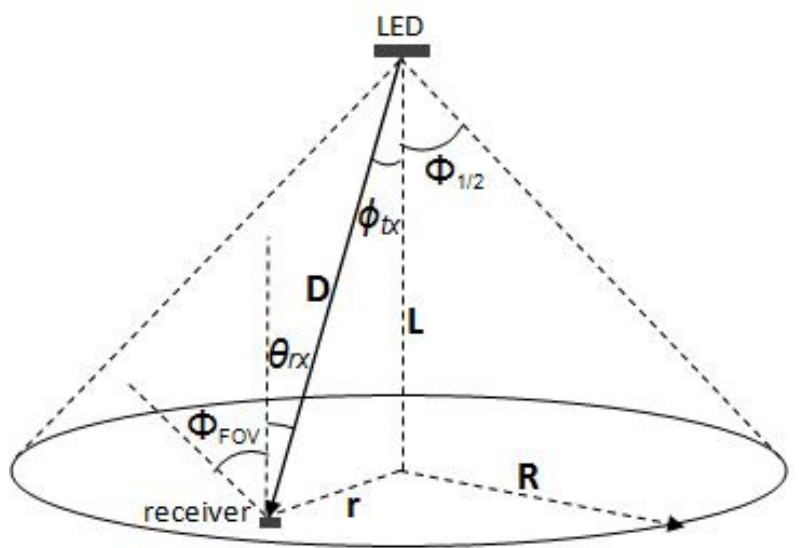

Fig. 1: Visible light communications system model

receiver designs and presents their performance. Section $\mathrm{V}$ discusses the numerical results and the paper is concluded in section VI.

\section{Channel and System Models}

We consider a SISO-VLC downlink transmission scenario, where an LED is mounted on the ceiling of a regular indoor environment to serve a user on the floor. The user is confined within a circular area ${ }^{1}$ with radius $R$ as shown in Fig. 1. The location of the user is assumed to be static, and a symbol-bysymbol detection is performed at the receiver side. However, extension of this work to include a coded system would be straightforward.

Considering that thermal noise and SDSN affect the optical signal $x$ sent from the LED, the received signal by the user can be written as

$$
y=h x+\sqrt{h x} n_{\mathrm{ds}}+n,
$$

where $x=s+d, s$ is the transmitted symbol and $d$ is the bias level (DC value). $n \sim \mathcal{N}\left(0, \sigma_{n}^{2}\right)$ is the signal-independent thermal noise where $\mathcal{N}\left(\mu, \sigma^{2}\right)$ represents a Gaussian distribution with mean $\mu$ and variance $\sigma^{2}$. The term $\sqrt{h x} n_{\mathrm{ds}}$ is the SDSN term, where $n_{\mathrm{ds}} \sim \mathcal{N}\left(0, \zeta^{2} \sigma_{n}^{2}\right)$. The term $\zeta^{2}$, which is the shot noise scaling factor that represents the strength of SDSN, is determined by the receiver parameters and occupies practical values ranging from 1 to 10 [30], [36]. However, our analysis is valid for any value of $\zeta^{2}$.

The DC offset value $d$ is added to guarantee that the VLC signal is always positive. Furthermore, $h$ denotes the DC channel gain of the VLC LoS beam between the LED and the photodetector, which can be expressed as [25], [37]

$$
h=\frac{A_{p d} \eta(m+1)}{2 \pi D^{2}} \cos ^{m}\left(\phi_{t x}\right) T_{s}\left(\theta_{r x}\right) g\left(\theta_{r x}\right) \cos \left(\theta_{r x}\right),
$$

where $A_{p d}$ is the detection area of the photodetector. Here, $\eta$ is the average responsivity of the receiver, $D$ is the Euclidean distance from the transmitter to the receiver, $\phi_{t x}$ and $\theta_{r x}$

\footnotetext{
${ }^{1}$ We assume that all users remain inside the circle. This is a practical assumption widely made in the literature; however, it is key to note that outside this region, there is no coverage by the specified LED.
}

refer to the angle of irradiance and the angle of incidence, respectively. $T_{S}\left(\theta_{r x}\right)$ is the gain of the receiver's optical filter, $m=\frac{-1}{\log _{2}\left(\cos \left(\Phi_{1 / 2}\right)\right)}$ is the Lambertian radiation order with LED emission semi-angle $\Phi_{1 / 2} \cdot g\left(\theta_{r x}\right)=n^{2} / \sin ^{2}\left(\Phi_{\mathrm{FOV}}\right)$ denotes the gain of the optical concentrator, which depends on the reflective index $n$ of the concentrator and the field-of-view angle $\Phi_{F O V}$. Note that $g\left(\theta_{r x}\right)=0$ when $\theta_{r x}>\Phi_{F O V}$.

Even though the VLC channel consists of both LoS and NLoS diffused signals caused by indoor reflectors, the weakest LoS signal is about $7 \mathrm{~dB}$ higher than the strongest NLoS signal in indoor environments [38], and thus, NLoS can be fairly neglected. Hence, we only consider the LoS VLC link in this work.

\section{Channel Estimation And Cramér-Rao lower BOUND}

Channel estimation is crucial for the performance of any wireless communication system, i.e., accurate estimation is essential for the follow-up equalization, demodulation, and decoding. Therefore, an accurate estimator leads to reliable communication systems [39].

In this section, we calculate the CRLB, which can be used as a benchmark to evaluate the estimators' performance in the presence of the SDSN. Then, least square (LS) and maximum likelihood (ML) estimators are presented and studied.

\section{A. Fisher Information and Cramér-Rao Lower Bound}

In this section, we find the Fisher information to calculate the CRLB. Let $\mathrm{N}$ be the number pilot symbols and $\mathbf{x}=\left[x_{1}, x_{2}, \cdots, x_{\mathrm{N}}\right]^{T}$ be the transmitted pilots' vector, where $(.)^{T}$ denotes the transpose operation. Then, the received signal vector $\mathbf{y}=\left[y_{1}, y_{2}, \ldots, y_{\mathrm{N}}\right]^{T}$ can be written as

$$
\mathbf{y}=h \mathbf{x}+\sqrt{h \operatorname{diag}(\mathbf{x})} \mathbf{n}_{d s}+\mathbf{n},
$$

where $\operatorname{diag}(\mathbf{x})$ is an $\mathrm{N} \times \mathrm{N}$ diagonal matrix with the elements of $\mathbf{x}$ in the main diagonal, $\mathbf{n}=\left[n_{1}, n_{2}, \ldots, n_{\mathrm{N}}\right]^{T}$, and $\mathbf{n}_{d s}=\left[n_{d s 1}, n_{d s 2}, \ldots, n_{d s \mathrm{~N}}\right]^{T}$. The elements of $\mathbf{n}$ are independent and identically distributed random variables, i.e., $\mathbf{n} \sim \mathcal{N}\left(0, \sigma_{n}^{2} I_{\mathrm{N}}\right)$ where $I_{\mathrm{N}}$ is the identity matrix of size $\mathrm{N}$. The same for $\mathbf{n}_{d s}$, i.e., $\mathbf{n}_{d s} \sim \mathcal{N}\left(0, \zeta^{2} \sigma_{n}^{2} I_{\mathrm{N}}\right)$. It is worth mentioning that all the pilot symbols must have positive values, i.e., $x_{i}>0$, where $i \in\{1,2, \cdots, \mathrm{N}\}$.

The lower bound on the variance of any unbiased estimator of $h$ can be obtained using the CRLB as follows ${ }^{2}$

$$
\sigma_{\hat{h}}^{2} \geq \frac{1}{\mathrm{~J}(h)}
$$

where $\mathrm{J}(h)$ is the Fisher information of $h$ given by

$$
\mathrm{J}(h)=-\mathbf{E}\left\{\frac{\partial^{2}}{\partial h^{2}} \ln f(\mathbf{y} ; h)\right\} .
$$

Here, $\mathbf{E}\{\cdot\}$ denotes the expectation operator and $\ln f(\mathbf{y} ; h)$ is the log-likelihood function of the unknown channel $h$. Since

\footnotetext{
${ }^{2}$ An estimator of a given parameter, e.g., $h$, is said to be unbiased if its expected value is equal to the true value of the parameter that should be estimated.
} 
we assume that all the samples are independent, the joint probability density function (PDF) of $\mathrm{N}$ observations, which is also equal to the likelihood function of $h, f(\mathbf{y} ; h)$, can be written as

$$
\begin{aligned}
& f(\mathbf{y} ; h)= \\
& \left(\prod_{i=1}^{N} \frac{1}{\sqrt{2 \pi \sigma_{n}^{2}\left(1+\zeta^{2} h x_{i}\right)}}\right) \exp \left(-\frac{1}{2 \sigma_{n}^{2}} \sum_{i=1}^{N} \frac{\left(y_{i}-h x_{i}\right)^{2}}{1+\zeta^{2} h x_{i}}\right) .
\end{aligned}
$$

After some tedious mathematical manipulation, $\mathrm{J}(h)$ can be expressed as

$$
\mathrm{J}(h)=\frac{1}{2} \sum_{i=1}^{N} \frac{\left(\zeta^{2} x_{i}\right)^{2}}{\left(1+\zeta^{2} h x_{i}\right)^{2}}+\frac{2 x_{i}^{2}}{\sigma_{n}^{2}\left(1+\zeta^{2} h x_{i}\right)} .
$$

Without loss of generality, and assuming that all the transmitted symbols are identical i.e., $x_{i}=p$, the Fisher information can be significantly simplified to

$$
\mathrm{J}(h)=\frac{N p^{2}}{2 \sigma_{n}^{2}} \frac{\zeta^{4} \sigma_{n}^{2}+2 \zeta^{2} h p+2}{\left(1+\zeta^{2} h p\right)^{2}} .
$$

Consequently, the CRLB can be expressed as

$$
\mathrm{CRLB}=\frac{2 \sigma_{n}^{2}}{N p^{2}} \frac{\left(1+\zeta^{2} h p\right)^{2}}{\zeta^{4} \sigma_{n}^{2}+2 \zeta^{2} h p+2} .
$$

It is observed from (9) that, as the number of pilots increases, the error estimation decreases. Furthermore, if $\zeta^{2} \rightarrow 0$, the CRLB reduces to

$$
\lim _{\zeta \rightarrow 0} \mathrm{CRLB}=\frac{\sigma_{n}^{2}}{N p^{2}} .
$$

This is a well-known result when there is no SDSN, and this validates the presented analysis. It is worth mentioning that the CRLB in (10) does not depend on the fading channel itself. However, as $\zeta \rightarrow \infty$, the CRLB in (9) can be expressed as

$$
\lim _{\zeta \rightarrow \infty} \mathrm{CRLB}=\frac{2 h^{2}}{N} .
$$

We can see that as $\zeta$ increases, the CRLB no longer depends on the transmitted power or the thermal noise. On the other hand, increasing the number of pilot symbols improves the CRLB; thus increasing the number of pilot symbols is more essential when trying to improve the CRLB than increasing the transmitted power in the presence of SDSN. Furthermore, as is evident from (11), as $\zeta$ increases, the CRLB starts to depend more on the fading channel $h$.

Remark 1: The CRLB performance does not have a linear relationship with $\zeta^{2}, \sigma_{n}^{2}$, or $h$. i.e., increasing $\zeta^{2}$ might increase or decrease the CRLB depending on $\sigma_{n}^{2}$ and $h$ values.

\section{B. Least Square Estimator}

Using the LS estimator, we can obtain a low-complexity receiver with no prior statistical information. The estimated $\hat{h}$ can be expressed as

$$
\hat{h}_{\mathrm{LS}}=\frac{\mathbf{x}^{T} \mathbf{y}}{\|\mathbf{x}\|^{2}}
$$

The performance of the LS estimator can be evaluated by determining the mean square error (MSE), which is equivalent to the variance of the estimation error. Thus, the MSE can be calculated as

$$
\epsilon_{\mathrm{LS}}=\hat{h}_{\mathrm{LS}}-h \text {. }
$$

From (12), $\hat{h}_{\mathrm{LS}}$ can be simplified to

$$
\hat{h}_{\mathrm{LS}}=h+\frac{\mathbf{x}^{T}}{\|\mathbf{x}\|^{2}} \sqrt{h \operatorname{diag}(\mathbf{x})} \mathbf{n}_{\mathrm{ds}}+\frac{\mathbf{x}^{T}}{\|\mathbf{x}\|^{2}} \mathbf{n} .
$$

Taking into consideration the fact that $\mathbf{E}\left\{\hat{h}_{\mathrm{LS}}\right\}=h$, as seen in (14), we can conclude that the LS estimator is unbiased. Furthermore, by applying both (13) and (14), $\epsilon_{\mathrm{LS}}$ can be written as

$$
\epsilon_{\mathrm{LS}}=\frac{\mathbf{x}^{T}}{\|\mathbf{x}\|^{2}}\left(\sqrt{h \operatorname{diag}(\mathbf{x})} \mathbf{n}_{\mathrm{ds}}+\mathbf{n}\right) .
$$

It is evident from (15) that $\epsilon_{\mathrm{LS}}$ is a Gaussian random variable, i.e., $\epsilon_{\mathrm{LS}} \sim \mathcal{N}\left(0, \sigma_{\epsilon_{\mathrm{LS}}}^{2}\right)$, where $\sigma_{\epsilon_{\mathrm{LS}}}^{2}$ is inversely proportional to the pilots' SNR value and can be written as

$$
\sigma_{\epsilon_{\mathrm{LS}}}^{2}=\frac{\sigma_{n}^{2} \sum_{i=1}^{N} x_{i}^{2}\left(1+\zeta^{2} h x_{i}\right)}{\left(\sum_{i=1}^{N} x_{i}^{2}\right)^{2}} .
$$

Now, assuming that the transmitted pilot $x_{i}=p$ for all $i, \sigma_{\epsilon_{L S}}^{2}$ can be further simplified as

$$
\sigma_{\epsilon_{L S}}^{2}=\frac{\sigma_{n}^{2}\left(1+\zeta^{2} h p\right)}{N p^{2}} .
$$

It can be noted from (17) that the obtained CRLB is smaller than the $\sigma_{\epsilon_{L S}}^{2}$, i.e.,

$$
\frac{\mathrm{CRLB}}{\sigma_{\epsilon_{L S}}^{2}}=\frac{2\left(1+\zeta^{2} h p\right)}{\zeta^{4} \sigma_{n}^{2}+2 \zeta^{2} h p+2}=\frac{2\left(1+\zeta^{2} h p\right)}{2\left(1+\zeta^{2} h p\right)+\zeta^{4} \sigma_{n}^{2}}<1 \text {. }
$$

Remark 2: The performance of the LS estimator reaches the CRLB in two cases: 1) when the transmitted energy $p \rightarrow \infty$, and 2) when $\zeta^{2} \rightarrow 0$.

\section{Maximum-Likelihood Estimator}

The ML estimator is a technique used to gauge unknown parameters by maximizing their likelihood PDF. Through the application this estimator, the log-likelihood of (6) can be given as

$$
\begin{aligned}
& \ln f(\mathbf{y} ; h)= \\
& -\frac{N}{2} \ln \left(2 \pi \sigma_{n}^{2}\right)-\frac{1}{2} \sum_{i=1}^{N}\left[\ln \left(1+\zeta^{2} h x_{i}\right)+\frac{\left(y_{i}-h x_{i}\right)^{2}}{\sigma_{n}^{2}\left(1+\zeta^{2} h x_{i}\right)}\right]
\end{aligned}
$$

The estimated channel in (19), can be determined by evaluating $\frac{\partial}{\partial h} \ln f(\mathbf{y} ; h)=0$, which can be expressed as

$$
\begin{aligned}
-\frac{1}{2} & \sum_{i=1}^{N} \frac{\zeta^{2} x_{i}}{1+\zeta^{2} h x_{i}} \\
& +\frac{1}{2 \sigma_{n}^{2}} \sum_{i=1}^{N}\left[\frac{2 x_{i}\left(y_{i}-h x_{i}\right)}{1+\zeta^{2} h x_{i}}+\frac{\zeta^{2} x_{i}\left(y_{i}-h x_{i}\right)^{2}}{\left(1+\zeta^{2} h x_{i}\right)^{2}}\right]=0 .
\end{aligned}
$$


TABLE I: Theoretical asymptotic and actual mean for ML estimator at different power levels, when $h=0.1$ and $\zeta^{2}=1$.

\begin{tabular}{c|cccc}
\hline Number of pilots, $N$ & $15 \mathrm{~dB}$ & $20 \mathrm{~dB}$ & $25 \mathrm{~dB}$ & $30 \mathrm{~dB}$ \\
\hline 1 & 0.0866 & 0.0952 & 0.0984 & 0.0995 \\
5 & 0.0974 & 0.0991 & 0.0997 & 0.0999 \\
10 & 0.0987 & 0.0995 & 0.0998 & 0.1 \\
15 & 0.0992 & 0.0997 & 0.0999 & 0.1 \\
25 & 0.0995 & 0.0998 & 0.0999 & 0.1 \\
50 & 0.0997 & 0.0999 & 0.1 & 0.1 \\
Asymptotic value & 0.1 & & & \\
\hline
\end{tabular}

While it is nearly impossible to manually find a closed-form expression for the roots of the above equation $\left(\hat{h}_{\mathrm{ML}}\right)$, numerical tools and software, such as Matlab, can easily solve for $\hat{h}_{\mathrm{ML}}$.

In order to simplify the analysis while maintaining a degree of generality, we assume that the transmitted pilot $x_{i}=p$. After some mathematical manipulations, which were realized by taking into account the fact that $h$ can never be negative, (20) can be expressed as the following quadratic equation

$$
-h^{2}+B h+C=0,
$$

where $B$ and $C$ can be expressed respectively as

$$
B=-\frac{2}{\zeta^{2} p}-\frac{\zeta^{2} \sigma_{n}^{2}}{p},
$$

and

$$
C=-\frac{\sigma_{n}^{2}}{p^{2}}+\frac{\sum_{i=1}^{N} y_{i}^{2}}{N p^{2}}+\frac{2 \sum_{i=1}^{N} y_{i}}{N \zeta^{2} p^{2}} .
$$

When determining the roots of the equation, keeping in mind that the VLC channel cannot be negative, we can obtain the following result

$$
\hat{h}_{\mathrm{ML}}=\frac{1}{2} B+\frac{1}{2} \sqrt{B^{2}+4 C} .
$$

It is clear here that the estimator is biased, i.e., $\mathbf{E}\left\{\hat{h}_{\mathrm{ML}}\right\} \neq h$. When considering the law of large numbers, however, it remains a reasonable estimator because as $N \rightarrow \infty$ and $\frac{1}{N} \sum_{i=1}^{N} y_{i}^{2} \rightarrow \mathbb{E}\left\{y_{i}^{2}\right\}=h^{2} p^{2}+\sigma_{n}^{2}\left(1+\zeta^{2} h p\right), \frac{1}{N} \sum_{i=1}^{N} y_{i} \rightarrow$ $\mathbb{E}\left\{y_{i}\right\}=h p$. Therefore, from (24) we obtain

$$
\begin{aligned}
& \mathbf{E}\left\{\hat{h}_{\mathrm{ML}}\right\}=\mathbf{E}\left\{\frac{1}{2}\left[-\frac{2}{\zeta^{2} p}-\frac{\zeta^{2} \sigma_{n}^{2}}{p}\right]\right. \\
& \left.+\frac{1}{2} \sqrt{\left[-\frac{2}{\zeta^{2} p}-\frac{\zeta^{2} \sigma_{n}^{2}}{p}\right]^{2}+4\left[-\frac{\sigma_{n}^{2}}{p^{2}}+\frac{\sum_{i=1}^{N} y_{i}^{2}}{N p^{2}}+\frac{2 \sum_{i=1}^{N} y_{i}}{N \zeta^{2} p^{2}}\right]}\right\} \\
& \stackrel{N \rightarrow \infty}{\longrightarrow} \frac{1}{2}\left[-\frac{2}{\zeta^{2} p}-\frac{\zeta^{2} \sigma_{n}^{2}}{p}\right] \\
& +\frac{1}{2} \sqrt{\left[-\frac{2}{\zeta^{2} p}-\frac{\zeta^{2} \sigma_{n}^{2}}{p}\right]^{2}+4\left[h^{2}+h\left(\frac{\zeta^{2} \sigma_{n}^{2}}{p}+\frac{2}{\zeta^{2} p}\right)\right]} \\
& =\frac{1}{2}\left[-\frac{2}{\zeta^{2} p}-\frac{\zeta^{2} \sigma_{n}^{2}}{p}\right]+\frac{1}{2} \sqrt{\left(2 h+\left[\frac{2}{\zeta^{2} p}+\frac{\zeta^{2} \sigma_{n}^{2}}{p}\right]\right)^{2}} \\
& =h .
\end{aligned}
$$

It should be noted here that in practice, it is seldom known in advance how large $N$ must be in order for (25) to hold,
TABLE II: Theoretical asymptotic and actual mean for ML estimator at different power levels, when $h=0.1$ and $\zeta^{2}=5$.

\begin{tabular}{c|cccc}
\hline Number of pilots, $N$ & $15 \mathrm{~dB}$ & $20 \mathrm{~dB}$ & $25 \mathrm{~dB}$ & $30 \mathrm{~dB}$ \\
\hline 1 & 0.0805 & 0.0847 & 0.0926 & 0.0976 \\
5 & 0.0951 & 0.0968 & 0.0986 & 0.0995 \\
10 & 0.0974 & 0.0984 & 0.0993 & 0.998 \\
15 & 0.0983 & 0.0989 & 0.0996 & 0.0998 \\
25 & 0.0990 & 0.0993 & 0.0997 & 0.999 \\
50 & 0.0995 & 0.0997 & 0.999 & 0.999 \\
Asymptotic value & 0.1 & & & \\
\hline
\end{tabular}

however, our simulation results show that, depending on $\zeta^{2}$, $N \in\{5,20\}$ is sufficiently enough to satisfy (25).

For the values $h=0.1$ and $\sigma^{2}=1$, the results are shown in Tables I and II for varying numbers of pilot symbols. It is evident from the tables that a greater number of samples is needed for the real value to converge at higher values of $\zeta^{2}$. It can also be observed that the mean converges around $N=5$, for $\zeta^{2}=1$, and $N=25$ for $\zeta^{2}=5$, at different levels of power.

Unlike the LS estimator, where the estimated $\hat{h}_{\text {LS }}$ is always a Gaussian random variable, it is obvious from (24) that $\hat{h}_{\mathrm{ML}}$ cannot be a Gaussian random variable so long as $\zeta \neq 0$. Thus, since the estimation is biased, the estimation error $\epsilon_{\mathrm{ML}}=h-$ $\hat{h}_{\mathrm{ML}}$ cannot be a Gaussian random variable and cannot have a zero mean. As a result, the MSE does not equal the variance of the error, $\epsilon_{\mathrm{ML}}$, but rather it equals $\mathbf{E}\left\{\epsilon_{\mathrm{ML}}^{2}\right\}-\left(\mathbf{E}\left\{\epsilon_{\mathrm{ML}}\right\}\right)^{2}$.

It is extremely difficult to analytically study the MSE of the ML estimation, so we must resort to some sort of approximation. The PDF, or histogram, of $\hat{h}_{\mathrm{ML}}$ was constructed using a Monte Carlo simulation in Fig. (2), and this was done for $N=5$. In the same figure, the theoretical PDF of $\mathcal{N}(h$, CRLB $)$ is plotted. It is evident from the figure that, when superimposed, the histogram and theoretical PDF can hardly be distinguished from each other. In other words, the theoretical PDF and histogram resemble each other to an almost identical degree. Note that, for $N=5$, the estimated PDF is slightly displaced to the left. This can also be observed in Tables I and II, where the expected value of the estimated $\hat{h}_{\mathrm{ML}}$ is to the left of the actual value of $h$. However, for large values of $N$, the estimated PDF is expected to more closely resemble the theoretical PDF.

We can conclude from the above discussion that, with a sufficient number of pilot symbols (around $N=5$ ), the ML is unbiased and, most importantly, achieves the CRLB, i.e., $\hat{h}_{\mathrm{ML}} \sim \mathcal{N}(h, \mathrm{CRLB})$. The ML estimator performance consequently reaches the CRLB. It is worth mentioning that LS estimator does not achieve CRLB with a small number of pilots, and this comparison demonstrates the superiority of ML over LS. On the other hand, LS proves to be less complex since the ML estimator requires information on $\sigma_{n}^{2}$ and $\zeta^{2}$.

\section{Discussions on the Results}

The previous section concluded that the ML estimator is a biased while the LS is not. Recall that the CRLB expresses the lower bound of the variance for the unbiased estimators of a deterministic parameter. 


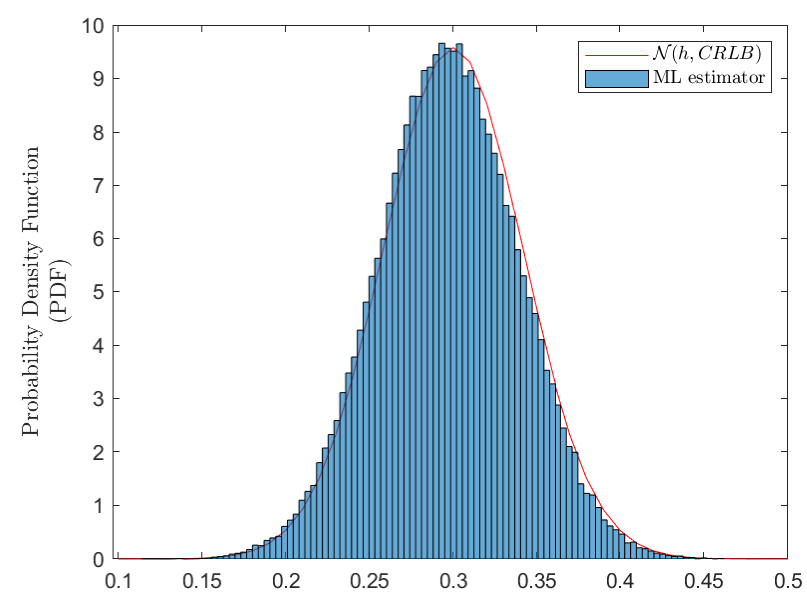

Fig. 2: The PDF of $\mathcal{N}(h, \mathrm{CRLB})$ and the histogram of the ML estimator when $N=5, \sigma_{n}^{2}=1$ and $h=0.3$.

Fig. 3 plots the CRLB against the LS and ML estimators' performance for one pilot when ${\sigma_{n}}^{2}=10^{-1}$ and $h=10^{-2}$ at different levels of SDSN. Various observations can be made, and they can all be summarized as follows:

- Both ML and LS estimators have the same performance as the CRLB when $\zeta^{2}=0$.

- The performance of the ML estimator eclipses that of the LS and CRLB when using one pilot.

- Increasing the SDSN level increases the difference between the estimators' performances. This can be clearly noticed from the subplots of $\zeta^{2}=3$ and $\zeta^{2}=10$.

- Increasing the SDSN level has a negative impact on the performance of the LS estimator.

- The relationship between the SDSN level and CRLB is non-linear, as can be concluded when looking at the CRLB subplot. Here, increasing the value of $\zeta^{2}$ has a positive effect before a threshold power value and a negative effect afterwards. The same trend can be seen for the ML estimator in the ML subplot.

- The SDSN has a notable impact on the channel estimation accuracy in the high-energy region.

- All curves approach the same performance after a certain energy value. This value shifts to the right at higher levels of SDSN.

Fig. 4 plots the CRLB against LS and ML estimators' performances while increasing the number of pilots. Here, $\sigma_{n}^{2}=1$, $h=10^{-1}$ and $\zeta^{2}=5$. Two conclusions can be drawn from this figure: 1) All the remarks made when discussing the previous figure remain valid when increasing the number of pilots and 2) the performance of the ML approaches that of the CRLB when increasing the number of pilots.

\section{Receiver Designs and Performance Analysis}

This section proposes two receiver designs, namely an optimal receiver and its less complex sub-optimal version. Both receivers are presented under two scenarios: perfect and

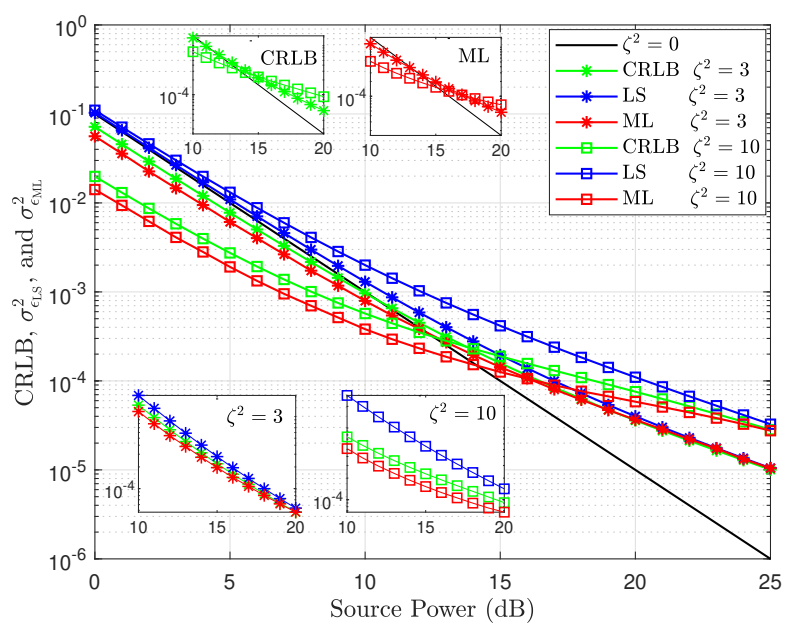

Fig. 3: CRLB, $\sigma_{\epsilon_{\mathrm{LS}}}^{2}$ and $\sigma_{\epsilon_{\mathrm{ML}}}^{2}$ when $\sigma_{n}^{2}=10^{-1}$ and $h=10^{-2}$.

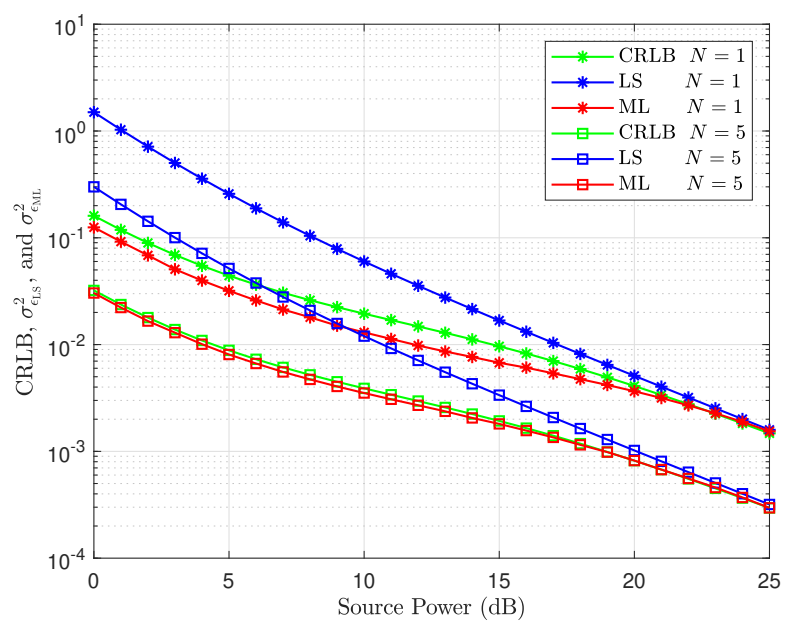

Fig. 4: CRLB, $\sigma_{\epsilon_{L S}}^{2}$, and $\sigma_{\epsilon_{\mathrm{ML}}}^{2}$ at different number of pilots when $\sigma_{n}^{2}=1$ and $h=10^{-1}$.

imperfect channel estimation. Furthermore, the error analysis of both receivers is presented in the context of on-off-keying (OOK) modulation.

\section{A. Receiver Designs}

Considering the discussed VLC wireless communication system, the SDSN changes the PDF of the received signal. There is a consequent need to design a receiver that can support the resulting PDF. It is assumed that the $M$-PAM modulation scheme is applied, and that $\left(x_{p}= \pm(2 b+1) \Delta+d\right)$ is the modulated baseband transmitted signal. Here, $b \in$ $\{0,1, \cdots, M-1\}, \Delta$ is the distance between the constellation points, and $d$ is the DC bias level that is strategically chosen in such a way that all the constellation points have positive values.

From (1), the joint PDF of the received signal $y$, given the 
transmitted signal $x_{p}$, can be expressed as

$$
f_{y \mid x_{p}}(y)=\frac{1}{\sqrt{2 \pi \sigma_{n}^{2}\left(h x_{p} \zeta^{2}+1\right)}} \times \exp \left\{-\frac{\left(y-h x_{p}\right)^{2}}{2 \sigma_{n}^{2}\left(h x_{p} \zeta^{2}+1\right)}\right\} .
$$

Assuming equal probability of all transmitted symbols, i.e., $p\left(x_{p}\right)=\frac{1}{M}$, the ML optimal receiver can be expressed as

$$
\hat{x}_{o p t}=\max _{x_{p}, p=0, \cdots, M-1}\left\{-\ln \left(h x_{p} \zeta^{2}+1\right)-\frac{\left(y-h x_{p}\right)^{2}}{\sigma_{n}^{2}\left(h x_{p} \zeta^{2}+1\right)}\right\} .
$$

Here, it can be concluded that the traditional ML receiver, which applies the minimum Euclidean distance (MED), is not the optimal receiver in the presence of SDSN. However, the traditional sub-optimal receiver that does not need any statistical information on shot and thermal noises can be written as

$$
\hat{x}_{s u b}=\min _{x_{p}, p=1, \cdots, M-1}\left\{\left(y-h x_{p}\right)^{2}\right\} .
$$

It will be shown later that both receivers have a comparable error performance in some cases. The same detection formulas in (27) and (28) can be applied in the case of imperfect CSI by replacing $h$ with $\hat{h}$.

OOK is the simplest method that can be used in VLC systems (IEEE 802.15.7). With this technique, the intensity of an optical source is directly modulated by the information sequences, which are usually binary. It is readily seen that OOK is a special case of $M$-PAM, where $x_{0}=0, x_{1}=2 A$ (the DC bias $d=A$ ). By applying the optimal receiver derived in (27), the optimal receiver for the OOK can be eventually formulated as

$$
\begin{aligned}
& \hat{x}_{o p t}= \\
& \begin{cases}x_{0} & \text { if } \frac{\zeta^{2}}{2} y^{2}+y<h A+\frac{\sigma_{n}^{2}}{4 A h}\left[1+2 A h \zeta^{2}\right] \ln \left[1+2 A h \zeta^{2}\right], \\
x_{1} & \text { otherwise. }\end{cases}
\end{aligned}
$$

Following the same logic, the sub-optimal receiver can be formulated as

$$
\hat{x}_{\text {sub }}= \begin{cases}x_{0} & \text { if } y \leq h A \\ x_{1} & \text { otherwise }\end{cases}
$$

In this case, the same detection formulas in (29) and (30) can be applied to the case of imperfect CSI if we replace $h$ with $\hat{h}$. It is worth mentioning that the sub-optimal receiver is identical to the optimal one in the absence of input-dependent noise.

\section{B. Error Analysis of Sub-Optimal Receiver}

In this subsection, the error analysis of the sub-optimal receiver is discussed. Given the received signal in (1), and by utilizing the $M$-PAM modulation, (1) can be rewritten as

$$
y=x_{p} h+\omega,
$$

where $\omega \sim \mathcal{N}\left(0, \sigma_{n}^{2}\left(1+x_{p} h \zeta^{2}\right)\right)$.
1) Perfect Estimation: The conditional probability (conditioned on the channel fading gain $h$ ) of using the sub-optimal receiver to detect $x_{q}$ instead of the transmitted $x_{p}$ can be written as

$$
\begin{aligned}
P\left(x_{p}, x_{q} \mid h\right) & =P\left(\left[y-h x_{p}\right]^{2}>\left[y-h x_{q}\right]^{2}\right) \\
& =P\left(2 h\left(x_{p}-x_{q}\right) \omega>h^{2}\left(x_{p}-x_{q}\right)^{2}\right) \\
& =Q\left(\sqrt{\frac{h^{2}\left(x_{p}-x_{q}\right)^{2}}{4 \sigma_{n}^{2}\left(1+h x_{p} \zeta^{2}\right)}}\right),
\end{aligned}
$$

where $P(\cdot)$ denotes the probability of an event. Now, the symbol error probability (SER) of the $M$-PAM VLC system can be represented as

$$
\begin{aligned}
P_{S}(e)=\frac{1}{M}( & P\left(x_{0}, x_{1}\right)+P\left(x_{M-1}, x_{M-2}\right) \\
& \left.+\sum_{i=p}^{M-1}\left[P\left(x_{i-1}, x_{i}\right)+P\left(x_{i-1}, x_{i-2}\right)\right]\right) .
\end{aligned}
$$

As a special case, the BER value for OOK can be written as

$$
P(e \mid h)=\frac{1}{2} P\left(x_{0}, x_{1} \mid h\right)+\frac{1}{2} P\left(x_{1}, x_{0} \mid h\right) .
$$

From (32), the BER in (34) can be expressed as

$$
P(e \mid h)=\frac{1}{2} Q\left(\sqrt{\frac{h^{2} A^{2}}{\sigma_{n}^{2}}}\right)+\frac{1}{2} Q\left(\sqrt{\frac{h^{2} A^{2}}{\sigma_{n}^{2}\left(1+2 A h \zeta^{2}\right)}}\right) .
$$

It is worth mentioning that the error probability of $P\left(x_{0}, x_{1} \mid h\right)<P\left(x_{1}, x_{0} \mid h\right)$, and therefore, the error probability is nonidentical to what is seen in traditional RF systems.

Moreover, in the absence of SDSN, i.e., $\zeta=0$, the above expression can be simplified to

$$
P(e \mid h)_{\zeta=0}=Q\left(\sqrt{\frac{h^{2} A^{2}}{\sigma_{n}^{2}}}\right) .
$$

Remark 3: According to (35) and (36), we have

$$
\frac{P(e \mid h)_{\zeta \neq 0}}{P(e \mid h)_{\zeta=0}}=\frac{1}{2}\left[1+\frac{Q\left(\sqrt{\frac{h^{2} A^{2}}{\sigma_{n}^{2}\left[1+2 A h \zeta^{2}\right]}}\right)}{Q\left(\sqrt{\frac{h^{2} A^{2}}{\sigma_{n}^{2}}}\right)}\right]>1,
$$

which is valid because the second term in the bracket is greater than one. Hence, as expected, the BER performance when $\zeta=$ 0 is better than what is seen when $\zeta \neq 0$. In other words, the SDSN deteriorates the error system performance. Furthermore, the asymptotic error performance as $\zeta \rightarrow \infty$, i.e., large values of $\zeta$, can be written as

$$
P(e \mid h)_{\zeta \rightarrow \infty}=\frac{1}{4}+\frac{1}{2} Q\left(\sqrt{\frac{h^{2} A^{2}}{\sigma_{n}^{2}}}\right) \approx \frac{1}{4}
$$

2) Imperfect Estimation: The receiver estimates the channel gain $h$ and uses the result in the same metric that would be 
applied if the channel was perfectly known. Hence, the conditional probability of detecting $x_{q}$ instead of the transmitted $x_{p}$ can be expressed as

$$
\begin{aligned}
P\left(x_{p}, x_{q} \mid \hat{h}\right) & =P\left(\left[y-\hat{h} x_{p}\right]^{2}>\left[y-\hat{h} x_{q}\right]^{2}\right) \\
& =Q\left(\sqrt{\frac{\hat{h}^{2}\left(x_{p}-x_{q}\right)^{2}}{4 \sigma_{n}^{2}\left(1+h x_{p} \zeta^{2}\right)+4 x_{p}^{2} \sigma_{\epsilon}^{2}}}\right),
\end{aligned}
$$

where $\epsilon$ denotes the channel estimation error. The SER of $M$ PAM can be obtained by substituting (39) into (33). Moreover, the OOK BER can be rewritten as

$$
P(e \mid \hat{h})=\frac{1}{2} Q\left(\sqrt{\frac{\hat{h}^{2} A^{2}}{\sigma_{n}^{2}}}\right)+\frac{1}{2} Q\left(\sqrt{\frac{\hat{h}^{2} A^{2}}{\sigma_{n}^{2}\left(1+2 A h \zeta^{2}\right)+4 A^{2} \sigma_{\epsilon}^{2}}}\right) .
$$

It is clear that the error performance degrades due to imperfect channel estimation.

\section{Error Analysis of the Optimal receiver for OOK}

In this subsection, the optimal error analysis for OOK is presented for both perfect and imperfect estimation cases. The error probability of $P\left(x_{1}, x_{0}\right)$ can be written as

$$
\begin{aligned}
& P\left(x_{1}, x_{0} \mid h\right) \\
& =P\left(\frac{\zeta^{2}}{2}(2 A h+\omega)^{2}+2 A h+\omega<h A+\frac{\sigma_{n}^{2}}{4 A h} \gamma \ln \gamma\right) \\
& =P\left(\frac{\zeta^{2}}{2 \sqrt{\gamma}} u^{2}+u<\frac{-h A}{\sqrt{\gamma}}+\frac{\sigma_{n}^{2}}{4 A h \sqrt{\gamma}} \ln \gamma\right),
\end{aligned}
$$

where $\gamma=1+2 A h \zeta^{2}$ and $u \sim \mathcal{N}\left(0, \sigma_{n}^{2}\right)$. To simplify the analysis, and for small values of $\sigma_{n}^{2}<0.001$, the above equation can be approximated as

$$
\begin{aligned}
P\left(x_{1}, x_{0} \mid h\right) & \approx P\left(u>\frac{h A+\frac{\zeta^{2} \sigma_{n}^{2}}{2}-\frac{\sigma_{n}^{2}}{4 A h} \ln \left(1+2 A h \zeta^{2}\right)}{\sqrt{1+2 A h \zeta^{2}}}\right) \\
& \approx Q\left(\sqrt{\frac{\left[h A+\frac{\zeta^{2} \sigma_{n}^{2}}{2}-\frac{\sigma_{n}^{2}}{4 A h} \ln \left(1+2 A h \zeta^{2}\right)\right]^{2}}{\sigma_{n}^{2}\left(1+2 A h \zeta^{2}\right)}}\right) .
\end{aligned}
$$

Furthermore, the error probability $P\left(x_{0}, x_{1} \mid h\right)$ can be given as

$$
\begin{aligned}
& P\left(x_{0}, x_{1} \mid h\right) \approx \\
& Q\left(\sqrt{\frac{\left[h A-\frac{\zeta^{2} \sigma_{n}^{2}}{2}+\frac{\sigma_{n}^{2}\left(1+2 A h \zeta^{2}\right)}{4 A h} \ln \left(1+2 A h \zeta^{2}\right)\right]^{2}}{\sigma_{n}^{2}}}\right) .
\end{aligned}
$$
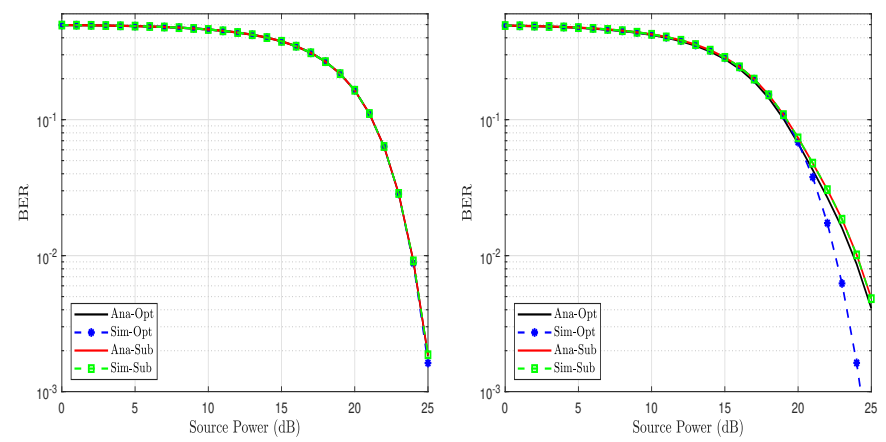

(a) $\sigma_{n}{ }^{2}=10^{-4}$ and $h=10^{-4}$.

(b) $\sigma_{n}^{2}=10^{-2}$ and $h=2 \times 10^{-3}$.

Fig. 5: Analytical and simulation BER performance for the optimal and sub-optimal receivers.

Therefore, the overall error probability can be expressed as

$$
\begin{aligned}
& P(e \mid h) \approx \frac{1}{2} Q\left(\sqrt{\frac{\left[h A+\frac{\zeta^{2} \sigma_{n}^{2}}{2}-\frac{\sigma_{n}^{2}}{4 A h} \ln \left(1+2 A h \zeta^{2}\right)\right]^{2}}{\sigma_{n}^{2}\left(1+2 A h \zeta^{2}\right)}}\right) \\
& +\frac{1}{2} Q\left(\sqrt{\frac{\left[h A-\frac{\zeta^{2} \sigma_{n}^{2}}{2}+\frac{\sigma_{n}^{2}\left(1+2 A h \zeta^{2}\right)}{4 A h} \ln \left(1+2 A h \zeta^{2}\right)\right]^{2}}{\sigma_{n}^{2}}}\right) .
\end{aligned}
$$

Similarly, the error probability for imperfect channel estimation can be expressed as

$$
\begin{aligned}
& P(e \mid h) \approx \\
& \frac{1}{2} Q\left(\sqrt{\frac{\left[\hat{h} A-\frac{\zeta^{2} \sigma_{n}^{2}}{2}+\frac{\sigma_{n}^{2}\left(1+2 A \hat{h} \zeta^{2}\right)}{4 A \hat{h}} \ln \left(1+2 A \hat{h} \zeta^{2}\right)\right]^{2}}{\sigma_{n}^{2}}}\right) \\
& +\frac{1}{2} Q\left(\sqrt{\frac{\left[\hat{h} A+\frac{\zeta^{2} \sigma_{n}^{2}\left(1+2 A h \zeta^{2}\right)}{2\left(1+2 A \hat{h} \zeta^{2}\right)}-\frac{\sigma_{n}^{2}}{4 A \hat{h}} \ln \left(1+2 A \hat{h} \zeta^{2}\right)\right]^{2}}{\sigma_{n}^{2}\left(1+2 A h \zeta^{2}\right)}}\right) .
\end{aligned}
$$

The SER of $M$-PAM can be obtained by deriving $P\left(x_{p}, x_{q} \mid h\right)$ and substituting it into (33) for perfect channel estimation. Similarly, by deriving $P\left(x_{p}, x_{q} \mid \hat{h}\right)$ and substituting it into (33), we can determine the SER of $M$-PAM for imperfect channel estimation. However, these give rise to mathematically complex processes, so we consider them for our future work.

\section{Discussions and Simulation Results}

This section discusses the numerical results of SISO-VLC performance in a system under the joint effects of SDSN and imperfect CSI. All results are analyzed for both the optimal and sub-optimal receivers. We assume OOK modulation technique with two transmitted signals $x_{0}=0, x_{1}=2 A$. The simulation results are obtained using Monte Carlo simulations using at least $10^{6}$ transmitted symbols for each figure. 


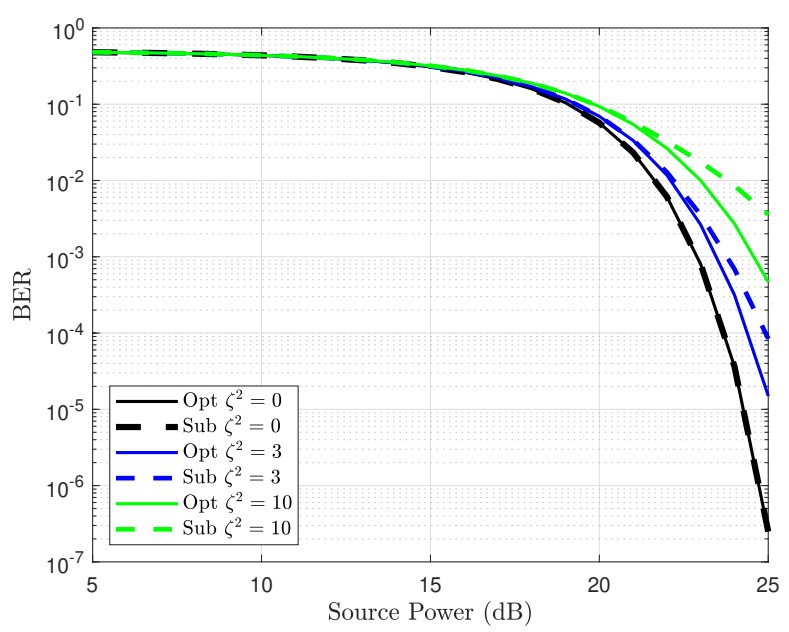

Fig. 6: BER performance at different levels of SDSN when $\sigma_{n}^{2}=10^{-3}$ and $h=5 \times 10^{-4}$.

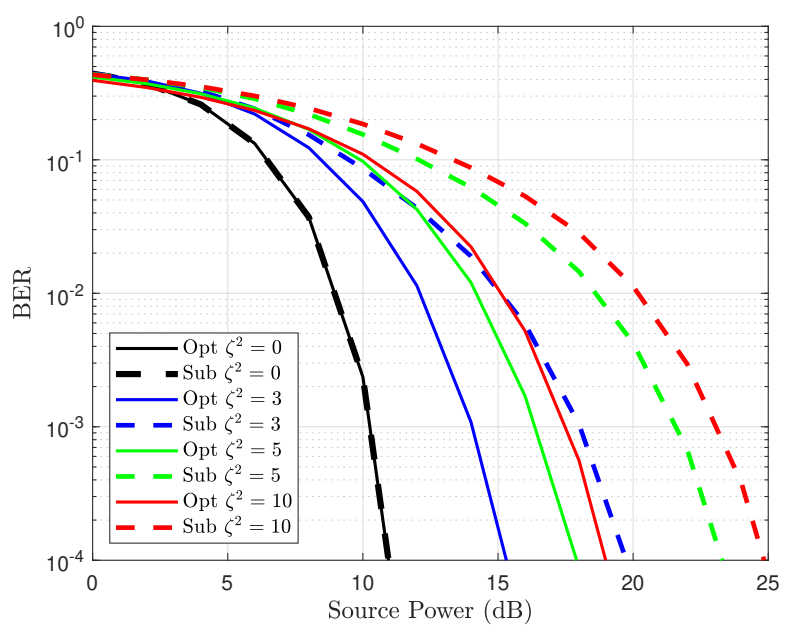

Fig. 7: BER performance at different levels of SDSN when $\sigma_{\epsilon}^{2}$ equals the CRLB with one pilot, $\sigma_{n}{ }^{2}=10^{-1}$ and $h=10^{-1}$.

Fig. 5 shows the analytical and simulation BER results for the optimal and sub-optimal receivers when $\zeta^{2}=5$ at different values of $\sigma_{n}^{2}$ and $h$. Three conclusions can be drawn from this figure: 1) In general, the optimal receiver outperforms the sub-optimal one, however, the performance difference between them shrinks as $\zeta^{2} \rightarrow 0$. 2) The analytical and simulation results of the sub-optimal receiver match perfectly for all values of $\sigma_{n}^{2}$, and finally 3) The analytical and simulation results of the optimal receiver perfectly match what is seen when $\sigma_{n}^{2}=10^{-4}$. We can consequently deduce that our approximation in (44) is fairly accurate, i.e., as long as $\sigma_{n}^{2} \leq 10^{-3}$.

Fig. 6 discusses the impact of $\zeta^{2}$ on the performance of the optimal and sub-optimal receivers when $\sigma_{n}{ }^{2}=10^{-3}$, $h=5 \times 10^{-4}$ and $\zeta^{2}$ is incremented along $[0,3,10]$. It can be observed that the BER increases as $\zeta^{2}$ increases. Moreover, the result here agrees with the one in the previous figure, where the optimal receiver outperforms the sub-optimal for

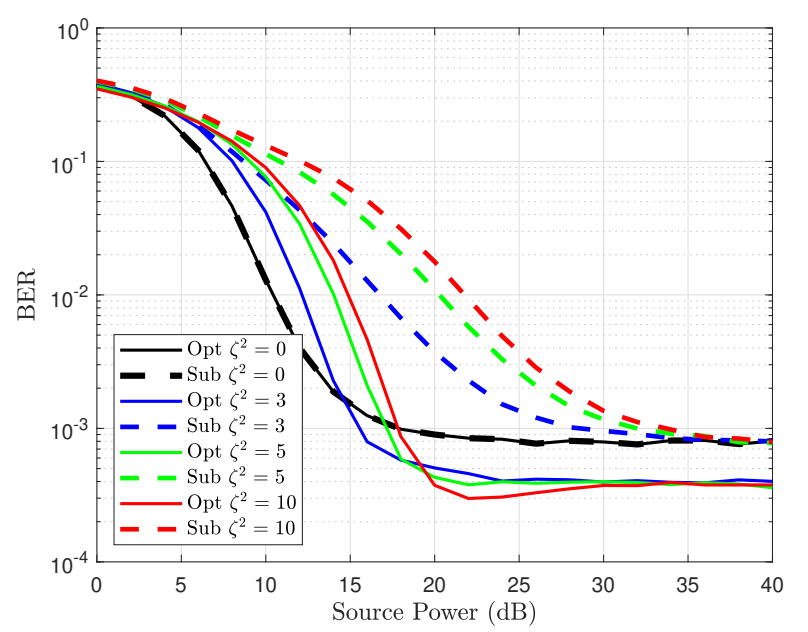

Fig. 8: BER performance at different levels of SDSN when $\sigma_{\epsilon}^{2}=10^{-3}, \sigma_{n}^{2}=10^{-1}$ and $h=10^{-1}$.

all values of $\zeta^{2}$. Until now, we have discussed the system performance assuming perfect CSI. In order to achieve more practical results, the joint impacts of imperfect CSI and the SDSN are analyzed in Figs. 7 and 8.

In Fig. 7, the effect of imperfect CSI is presented when the estimation error variance equals to the CRLB $\left(\sigma_{\epsilon}^{2}=\mathrm{CRLB}\right)$ upon the use of one pilot. As indicated by the figure, more degradation in the performance can be noticed due to the joint effects of the channel estimation error and the SDSN. This degradation worsens at higher levels of SDSN.

The effect of imperfect CSI when the estimation error variance has a fixed value of $\left(\sigma_{\epsilon}^{2}=10^{-3}\right)$ is given in Fig. 8 . As the figure shows, more degradation in the performance can be noticed compared to what was seen in Fig. 7 when the estimation error variance reached the CRLB. This confirms the importance of the estimation phase when trying to maintain the system performance. One more interesting observation related to the system performance in the high SNR region can be made: The performance of the sub-optimal receiver at different levels of SDSN saturates and reaches the performance of the optimal receiver when $\zeta^{2}=0$. This can be explained by noting the BER in (40) and (45), where $B E R \rightarrow Q\left(\sqrt{\frac{\hat{h}^{2}}{4 \sigma_{\epsilon}^{2}}}\right)$ as $A \rightarrow \infty$. One last conclusion that can be drawn is that the error performance of the optimal detector saturates at a lower error probability compared to the sub-optimal detector, i.e., the optimal detector saturates at $\mathrm{BER} \approx 2 \times 10^{-4}$ while the sub-optimal saturates at $\approx 1 \times 10^{-3}$.

\section{CONCLUSIONS}

This paper introduces an analytical framework to study the performance of SISO-VLC under the effect of SDSN in cases perfect and imperfect channel estimation. LS and ML estimators were proposed and compared to the derived CRLB. Furthermore, optimal and sub-optimal receivers were designed and compared. The analytical results corroborated by Monte Carlo simulation show the superiority of the optimal receiver design compared to the sub-optimal one in all conditions. 
In addition, results demonstrated that SDSN negatively affects the system BER and the channel estimation error bound, and that it has a non linear effect on CRLB. Closedform expressions of BER for sub-optimal detectors with OOK and $M$-PAM modulation techniques are derived, and an approximated BER expression for the optimal detector using OOK is presented. Deriving a closed-form expression of the BER for optimal receiver using OOK and $M$-PAM modulation techniques are considered for our future work.

\section{REFERENCES}

[1] P. H. Pathak, X. Feng, P. Hu, and P. Mohapatra, "Visible light communication, networking, and sensing: A survey, potential and challenges," IEEE Commun. Surveys Tuts, vol. 17, no. 4, pp. 2047-2077, 2015.

[2] S. Ma, H. Li, Y. He, R. Yang, S. Lu, W. Cao, and S. Li, "Capacity bounds and interference management for interference channel in visible light communication networks," IEEE Trans. Wireless Commun., vol. 18, no. 1, pp. 182-193, 2019

[3] J. Wang, J. Wang, B. Zhu, M. Lin, Y. Wu, Y. Wang, and M. Chen, "Improvement of BER performance by tilting receiver plane for indoor visible light communications with input-dependent noise," in 2017 IEEE Int. Conf. Commun. (ICC), 2017, pp. 1-6.

[4] A. Jovicic, J. Li, and T. Richardson, "Visible light communication: opportunities, challenges and the path to market," IEEE Commun. Mag., vol. 51, no. 12, pp. 26-32, 2013.

[5] R. Wang, Q. Gao, J. You, E. Liu, P. Wang, Z. Xu, and Y. Hua, "Linear transceiver designs for MIMO indoor visible light communications under lighting constraints," IEEE Trans. Commun., vol. 65, no. 6, pp. 2494 2508, 2017.

[6] Z. Wang, Q. Wang, and Z. Xu, Visible Light Communications: Modulation and Signal Processing. Hoboken, NJ, USA: Wiley, Nov 2017.

[7] J. Armstrong, "OFDM for optical communications," Journal of Lightwave Technology, vol. 27, no. 3, pp. 189-204, 2009.

[8] H. Elgala, R. Mesleh, and H. Haas, "Indoor broadcasting via white LEDs and OFDM," IEEE Trans. Consum. Electron., vol. 55, no. 3, pp. $1127-1134,2009$.

[9] X. Li, J. Vucic, V. Jungnickel, and J. Armstrong, "On the capacity of intensity-modulated direct-detection systems and the information rate of ACO-OFDM for indoor optical wireless applications," IEEE Trans. Commun., vol. 60, no. 3, pp. 799-809, 2012.

[10] W. O. Popoola, E. Poves, and H. Haas, "Error performance of generalised space shift keying for indoor visible light communications," IEEE Trans. Commun., vol. 61, no. 5, pp. 1968-1976, 2013.

[11] J. Wang, H. Ge, J. Zhu, J. Wang, J. Dai, and M. Lin, "Adaptive spatial modulation for visible light communications with an arbitrary number of transmitters," IEEE Access, vol. 6, pp. 37 108-37 123, 2018.

[12] Q. Gao, J. H. Manton, G. Chen, and Y. Hua, "Constellation design for a multicarrier optical wireless communication channel," IEEE Trans. Commun., vol. 62, no. 1, pp. 214-225, 2014.

[13] M. Beko and R. Dinis, "Systematic method for designing constellations for intensity-modulated optical systems," J. Opt. Commun. Netw., vol. 6 , no. 5, pp. 449-458, 2014

[14] C. Gong, S. Li, Q. Gao, and Z. Xu, "Power and rate optimization for visible light communication system with lighting constraints," IEEE Trans. Signal Process., vol. 63, no. 16, pp. 4245-4256, Aug. 2015.

[15] H. S. Khallaf, A. S. Ghazy, H. M. H. Shalaby, and S. S. A. Obayya, "Performance analysis of visible light communication systems over fading channels," in 19th Int. Conf. Transparent Opt. Netw.(ICTON), July 2017, pp. 1-4.

[16] Y. S. Hussein, M. Y. Alias, and A. A. Abdulkafi, "On performance analysis of LS and MMSE for channel estimation in VLC systems," in 2016 IEEE 12th International Colloquium on Signal Processing \& Its Applications (CSPA), 2016, pp. 204-209.

[17] X. Chen and M. Jiang, "Enhanced bayesian MMSE channel estimation for visible light communication," in 2016 IEEE 27th Annual International Symposium on Personal, Indoor, and Mobile Radio Communications (PIMRC), 2016, pp. 1-6.

[18] H. Dogan, O. Şaylı, and E. Panayirci, "Pilot assisted channel estimation for asymmetrically clipped optical OFDM over visible light channels," in 2016 IEEE International Black Sea Conference on Communications and Networking (BlackSeaCom), 2016, pp. 1-4.
[19] X. Chen and M. Jiang, "Adaptive statistical bayesian mmse channel estimation for visible light communication," IEEE Transactions on Signal Processing, vol. 65, no. 5, pp. 1287-1299, 2017.

[20] A. Yesilkaya, O. Karatalay, A. S. Ogrenci, and E. Panayirci, "Channel estimation for visible light communications using neural networks," in 2016 International Joint Conference on Neural Networks (IJCNN), 2016, pp. $320-325$.

[21] V. B. Manur and L. Ali, "MMSE based compressed sensing algorithms for channel estimation in VLC," in 2019 4th International Conference on Electrical, Electronics, Communication, Computer Technologies and Optimization Techniques (ICEECCOT), 2019, pp. 170-173.

[22] J. C. Estrada-Jiménez, B. G. Guzmán, M. J. Fernández-Getino García, and V. P. G. Jiménez, "Superimposed training-based channel estimation for MISO optical-OFDM VLC," IEEE Transactions on Vehicular Technology, vol. 68, no. 6, pp. 6161-6166, 2019.

[23] S. Ho, A. A. Saed, L. Lai, and C. W. Sung, "Coding and bounds for channel estimation in visible light communications and positioning," IEEE J. Selected Areas in Commun., vol. 36, no. 1, pp. 34-44, 2018.

[24] D. R. Ashok and A. Chockalingam, "Compact optimal pilot design for channel estimation in MIMO VLC systems," in 2019 IEEE Wireless Commun. and Netw. Conf. (WCNC), 2019, pp. 1-7.

[25] L. Yin, W. O. Popoola, X. Wu, and H. Haas, "Performance evaluation of non-orthogonal multiple access in visible light communication," IEEE Transactions on Communications, vol. 64, no. 12, pp. 5162-5175, 2016.

[26] S. Mohapatra, G. Satapathy, S. P. Dash, and P. R. Sahu, "Performance analysis of visible light communication system with imperfect CSI," IEEE Communications Letters, vol. 24, no. 12, pp. 2844-2848, 2020.

[27] Q. Gao, K. Qaraqe, and E. Serpedin, "Rotated color shift keying for visible light communications with signal-dependent noise," IEEE Cотти. Lett., vol. 24, no. 4, pp. 844-848, 2020.

[28] Q. Gao, K. Qaraqe, and E. Serpedin, "Improving the modulation designs for visible light communications with signal-dependent noise," IEEE Commun. Mag., vol. 58, no. 5, pp. 26-32, 2020.

[29] M. Safari, "Efficient optical wireless communication in the presence of signal-dependent noise," in 2015 IEEE International Conference on Communication Workshop (ICCW), 2015, pp. 1387-1391.

[30] H. Chen and Z. Xu, "A two-dimensional constellation design method for visible light communications with signal-dependent shot noise," IEEE Commun. Lett., vol. 22, no. 9, pp. 1786-1789, 2018.

[31] Q. Gao, S. Hu, C. Gong, and Z. Xu, "Modulation designs for visible light communications with signal-dependent noise," Journal of Lightwave Technology, vol. 34, no. 23, pp. 5516-5525, 2016.

[32] J. Wang, Z. Yang, Y. Wang, and M. Chen, "On the performance of spatial modulation-based optical wireless communications," IEEE Photonics Technology Lett., vol. 28, no. 19, pp. 2094-2097, 2016.

[33] Q. Gao, C. Gong, and Z. Xu, "Joint transceiver and offset design for visible light communications with input-dependent shot noise," IEEE Trans. Wireless Commun., vol. 16, no. 5, pp. 2736-2747, 2017.

[34] S. H. Lin, C. Liu, X. Bao, and J. Y. Wang, "Indoor visible light communications: performance evaluation and optimization," $J$ Wireless Com Network, vol. 228, pp. 1-12, 2018.

[35] J. Y. Wang, X. T. Fu, R. R. Lu, J. B. Wang, M. Lin, and J. Cheng, "Tight capacity bounds for indoor visible light communications with signal-dependent noise," IEEE Trans. on Wireless Commun., pp. 1-1, 2020.

[36] S. M. Moser, "Capacity results of an optical intensity channel with inputdependent gaussian noise," IEEE Trans Inf. Theory, vol. 58, no. 1, pp. 207-223, Jan 2012.

[37] Z. Ghassemlooy, W. Popoola, and S. Rajbhandari, Optical wireless communications: system and channel modeling with MATLAB, 1st ed. CRC Press, May 2017.

[38] L. Z. et al., "High data rate multiple input multiple output (MIMO) optical wireless communications using white led lighting," IEEE J. Sel. Areas Commun., vol. 27, no. 9, pp. 1654-1662, Dec. 2009.

[39] T. Wang, A. Hussain, Y. Cao, and S. Gulomjon, "An improved channel estimation technique for IEEE $802.11 \mathrm{p}$ standard in vehicular communications," Sensors, vol. 19, no. 1, p. 98, Jan. 2019. 